\title{
Age-associated gut microbiota impairs hippocampus-dependent memory in a vagus- dependent manner
}

\author{
Damien Rei, ${ }^{1}$ Soham Saha, ${ }^{1}$ Marianne Haddad, ${ }^{1}$ Anna Haider Rubio, ${ }^{1}$ Marie-Noelle \\ Ungeheuer ${ }^{2}$, Harry Sokol ${ }^{3}$ and Pierre-Marie Lledo ${ }^{1}$
}

1 Laboratory for Perception and Memory, Institut Pasteur, CNRS UMR 3571, 75015 Paris, France.

2 Investigation and Access to BioResources (ICAReB) plateform, 75015 Paris, France.

3 Gastroenterology Department, Sorbonne Université, INSERM, Centre de Recherche SaintAntoine, CRSA, AP-HP, Saint Antoine Hospital, Paris, France.

INRA, UMR1319 Micalis \& AgroParisTech, Jouy en Josas, France.

Paris Center for Microbiome Medicine, Fédération Hospitalo-Universitaire, Paris, France.

\section{Correspondence}

damien.rei@pasteur.fr

pierre-marie.lledo@pasteur.fr

\section{Conflict of interest statement}

The authors have declared that no conflict of interest exists.

\begin{abstract}
Aging is known to be associated with hippocampus-dependent memory decline, but the underlying causes of this age-related memory impairment are not yet elucidated. Here we show that the colonization of mice with the gut microbiota from aged, but not young animals is sufficient to trigger profound hippocampal alterations including astrogliosis, decreased adult neurogenesis, decreased novelty-induced neuronal activation and impairment in hippocampal-dependent memory. Similar alterations were reported in mice following the transfer of microbiota from aged human healthy donors. To decipher the mechanisms involved in mediating these microbiota-induced effects on brain functioning, we mapped the neuronal activity patterns and report that aged-microbiota transplantation reduced neuronal activity upstream to the vagus nerve. Targeted pharmacogenetic manipulation of the ascending branch of the vagus nerve demonstrated that the mere decrease in vagal activity was also detrimental to hippocampal functions. In contrast, increasing vagal activity alleviated the adverse effects of age-associated microbiota transfer on hippocampal functions and reinstated normal hippocampal memory in aged mice. We conclude that vagus nerve stimulation is a potential therapeutic strategy to lessen microbiota-dependent age-associated impairments in hippocampal functions.
\end{abstract}




\section{Graphical abstract}

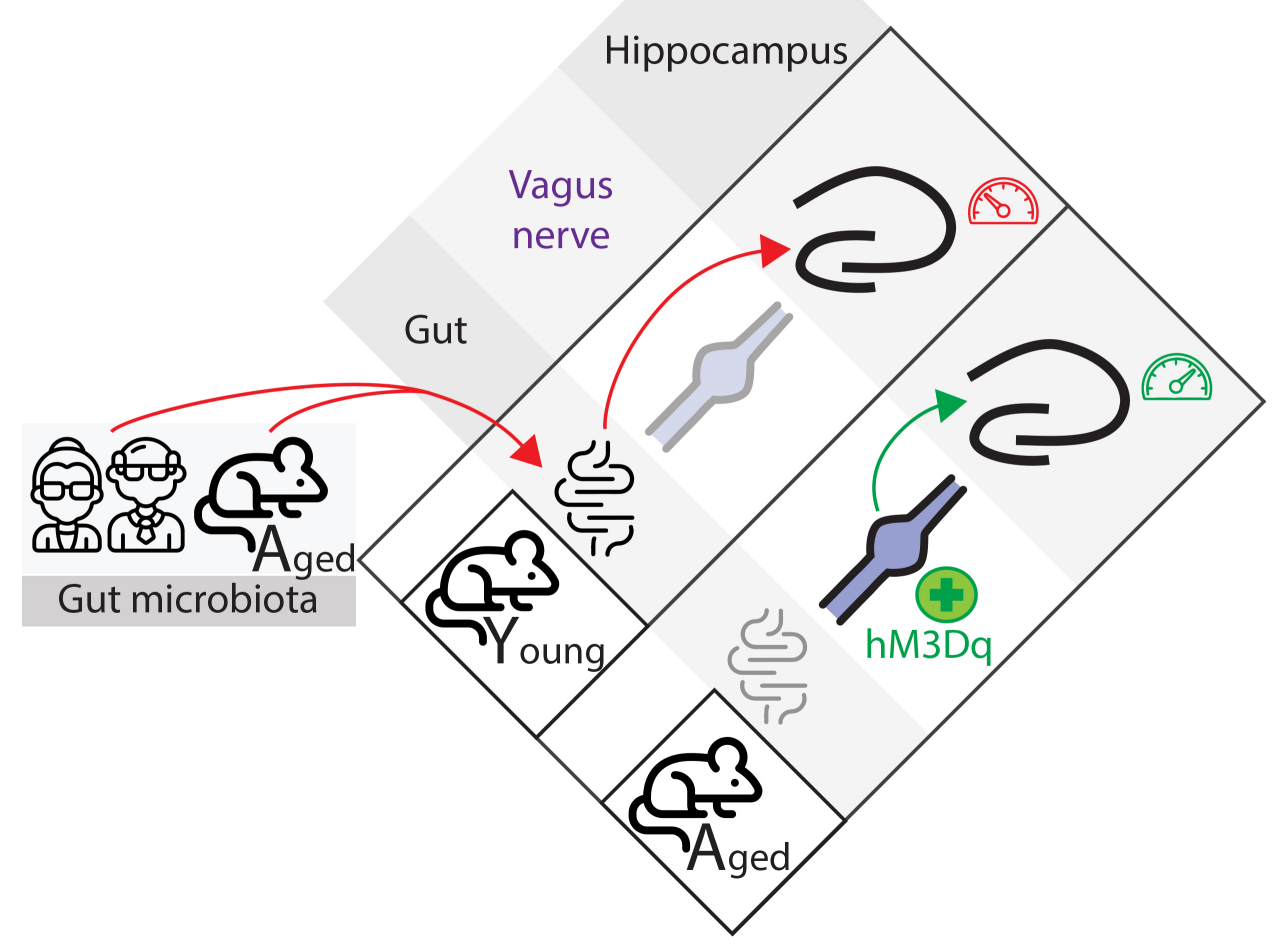




\section{Introduction}

The gut microbiota (GM) - the intestinal community of microorganisms - recently emerged as a key player for developing and maintaining homeostasis, particularly for the brain (reviewed in ${ }^{1}$ ).

In the brain, GM have been shown to modulate multiple functions, notably learning and memory. Thus, the GM can influence hippocampal functions such as adult neurogenesis ${ }^{5-}$ 7 , astrocyte function ${ }^{8}$ as well as systemic and brain inflammation ${ }^{9,10}$. When altered, these brain processes, frequently associated with aging, may ultimately lead to hippocampaldependent impairment in episodic and spatial memory ${ }^{11}$. However, understanding if the GM can autonomously influence aging, especially in humans, is challenging, since age-related changes in host physiology can influence the microbiota composition observed in aging and vice versa. So, it remains unknown to what extent gut microbes contribute to the aging phenotype, and if they do, what are the mechanisms at play.

Evidence is starting to uncover the causal role of GM changes in cognitive functions. In recent studies, GM, from aged mice or rats, transplanted to young counterparts altered learning and memory performances in the Barnes maze ${ }^{12}$ or delayed matching to position tests $^{9}$, respectively, while it promoted systemic and hippocampal inflammation which was associated with perturbation in the expression levels of synaptic plasticity genes.

The GM is known to directly communicate with the brain, notably through the vagus nerve (VN). The VN circuit is the most direct and well-studied neuronal pathway between the gut and the CNS (reviewed in ${ }^{13}$ ). VN sensory fibers innervate the muscular and mucosa layers of the gastrointestinal tract, detect mechanosensory and chemical signals and then relay these signals to the CNS through the ventro-medial part of the nucleus tractus solitarius (vmNTS) in the caudal brainstem ${ }^{14}$. This vagal gut-brain circuit, is also known to be linked to the hippocampus (HPC). The antidepressant effect of probiotic treatment on the hippocampal expression levels of GABA receptors was shown to require an intact $\mathrm{VN}$ and this effect was occluded in vagotomized animals ${ }^{2}$. Furthermore, VN electrical stimulation is an approved therapy for depression and drug-resistant temporal epilepsy (reviewed in ${ }^{15}$ ), and is known to be a powerful modulator of hippocampal functions, notably at the electrophysiological ${ }^{16}$ and epigenetic ${ }^{17}$ levels.

However, beyond rodent GM data, the effect of human age-associated GM on hippocampal memory is currently unknown. Here, we report that the transfer of ageassociated GM of both mouse and human origin to young mice mimicked some aspects of the deleterious impact of aging on the hippocampus, with a deficit in memory and inability of the hippocampal CA1 network to respond to a novel environment. The detrimental effect of ageassociated GM was also seen upstream to the VN. Targeted pharmacogenetic manipulation of $\mathrm{VN}$ activity uncover the role of $\mathrm{VN}$ tone on hippocampal function and its therapeutical impact on aging-related hippocampal dysfunction.

\section{Combined Results and Discussion}

\section{Age-associated GM of both murine and human origin impairs hippocampal memory and related markers}

To investigate the contribution of the transplanted GM in the detrimental impact of aging on hippocampal functions, young $(\mathbf{Y})$ adult mice (2 months old, thereafter referred to as young mice) were treated by broad-spectrum antibiotics, followed by sequential fecal microbiota transfer (FMT) to ensure GM-colonization from either the same age- (Young mice GM in 
Young mice $=$ Yin $Y)$ or aged $(\mathbf{A})-(18$ months old $)($ Aged mice $\mathrm{GM}$ in young mice $=$ AinY $)$ donor mice (Fig. 1A).

Analysis of the microbial composition in the donor and FMT-colonized mice was performed using 16S rRNA gene sequencing. Changes in the relative abundance of bacteria in both donor and recipient mice were observed at the taxonomic level of family (Fig. S1-1A), with a trend toward an age-associated increase in the Firmicutes to Bacteroides ratio that was no longer seen after the colonization (Fig. S1-1B). Alpha diversity is often used to measure the richness and uniformity of species in community ecology. Using Shannon, Simpson or Abundance-based Coverage Estimator (ACE) indexes, we did not observe any significant differences between the studied groups (Fig. S1-1C-E). Determination of the beta diversity, the comparative analysis of the composition of the microbial community in different samples, showed differences in the GM from young and old donors (clusters $Y$ and A, Fig. S1-1F) and recipient young mice receiving young- or aged-derived microbiota (clusters YinY and AinY, Fig. S1-1F). This result was supported by the lower Jaccard distance between donor and recipient mice than between $Y$ and $A$ or YinY and AinY mice (Fig. S1-1G). Although such changes were relatively subtle compared to previous studies using different mouse strains ${ }^{18-21}$, comparative analysis of the young and aged mice and transferred animals showed a clustering due to the age that was transferred to the colonized animals. We conclude that our FMT-transfer model caused successful transfer of GM "aging" characteristics to the recipient mice.

Next, the repercussion of GM colonization on the hippocampus was investigated at the behavioral and cellular level and compared to the same variables studied in aged mice. Hippocampus-dependent memory abilities was first scored before and after GM-colonization using the hippocampus-dependent isotropic version of the novel object location task ${ }^{22,23}, \mathrm{a}$ task known to be impacted in aged mice ${ }^{24}$. In this assay, mice explore two different objects they were previously familiarized with, and one object is displaced from its previous familiar location. Young mice before FMT showed normal discrimination memory performances in the test and this performance level was maintained following GM-transfer from the same agemice. Conversely, aged mice GM-transfer in young mice led to a deterioration of the recipient's memory to a level similar to aged mice (Fig. 1B). The detrimental impact of the aged mice-GM on young recipient mice memory was not specific to this task, as memory deficits were also observed in two other hippocampus-dependent behaviors, i.e., the novel object recognition ${ }^{24}$, and the contextual version of the fear conditioning ${ }^{25}$ (Fig. S1-2A-C).

To examine the impact of the age-associated GM on the hippocampus network, neuronal activity in dorsal hippocampal CA1 was monitored following exposure to novelty ${ }^{26,27}$. Mice were placed in a novel environment, whereas control mice were left undisturbed in their home cages (Fig. 1C schematics). The increase in the expression of the immediate early gene cFos following novelty exposure was performed by automated quantification in the hippocampal CA1. Novelty-induced upregulation of cFos in the dorsal CA1 was observed in animals transferred with young GM, whereas animals transferred with an aged microbiota showed reduced activation similar to that of aged mice (Fig. 1C). At the cellular level, we analyzed hippocampal astrogliosis and adult neurogenesis, two processes sensitive to aging ${ }^{28}$ and dependent on GM composition ${ }^{29}$. Age-associated GM transfer led to an increase in astrogliosis which was monitored using several criterions based on the astroglial marker GFAP, including its relative expression level in this cell population, cell size and number of branches (Fig. S1-2D). Age-associated GM transfer also led to a significant decrease in adult hippocampal neurogenesis, characterized by a reduction in the number of DCX-expressing immature adult-born neurons in the dentate gyrus (DG) (Fig. S1-2E). Thus, 
age-associated GM transfer in the colonized young animals impaired hippocampal memory, the ability of the CA1 network to respond to novelty exposure, promoted astrogliosis and decreased the number of newly-generated neurons, mimicking some of the hallmarks of aging on hippocampal structure and function.

We then sought to investigate whether the transfer of human gut microbiota could promote similar changes in the hippocampus of recipient mice. Fecal samples were collected from adult healthy human donors selected to be either, less than 35, or more than 65 years old. This material was used to perform FMT to young mice to evaluate the potential effects on memory using the ISO-NOL task (Fig. 1D). Mice transferred with each of the 4 young human-GM performed normally in the task. In contrast, the transfer of microbiota from the 4 aged human donors impaired hippocampus-dependent memory (Fig. 1E) and altered the novelty-induced CA1 activation (Fig. $1 \mathrm{~F}$ and S1-2F). We conclude that human microbiota transfer from old, but not young, human donors alters hippocampal function in recipient mice.

\section{Decrease in vagus nerve activity is both necessary and sufficient for age-associated GM impact on the hippocampus}

We next investigated whether the deleterious impact of age-associated GM transfer on young mice depends on VN signaling. To do so, the cFos-based activity level was assessed in the vmNTS, the entry point of VN ascending signaling into the brain (schematic Fig. 2A), which was taken as a proxy of VN ascending activity level. Automated quantification of the number of cFos+ cells in the vmNTS was reduced in mice transferred with an age-associated GM compared to their young-GM transferred counterparts (Fig. 2A). Next, to directly test the possible implication of $\mathrm{VN}$ activity for hippocampal memory function, we aimed at manipulating $\mathrm{VN}$ ascending activity by the viral transduction of the designer receptors exclusively activated by a designer drug (DREADD) in the left nodose ganglia (L-NG), to be consistent with the side used for electrical VN stimulation as a treatment for resistant major depression ${ }^{30}$. Co-injection of AAV1-hSyn-Cre and AAV5-hSyn-DIO-hM3Dq-mCherry efficiently labelled L-NG neurons soma and their axonal terminals could be seen in the ventromedial areas of the NTS (i.e., vmNTS; Fig. 2B). Three weeks after the viral delivery, the DREADD activator clozapine $\mathrm{N}$-oxide (CNO) was administered to induce DREADD activation (Fig. S2A). Using the excitatory form of DREADD, we confirmed that CNO injection significantly increased the number of cFos+ neurons in the vmNTS (Fig. S2B-C). Furthermore, when co-expressing both excitatory and inhibitory DREADD, we confirmed that the inhibitory DREADD hM4Di in the L-NG could block such CNO-induced increase in vmNTS cFos expression (Fig. S2B-C).

We next evaluated the hippocampal memory strength following a transient inhibition of the vagal ascending activity in an attempt to mimic the age-associated GM effect on ascending vagal signaling. The L-NG of young mice was transduced to express the inhibitory DREADD (or mCherry only for control). Three weeks later, animals received a CNO i.p. injection to induce L-NG inhibition during the ISO-NOL test (Fig. 2C). This treatment completely impaired their discrimination ability in the task (Fig. 2D) and this DREADD-induced loss of performance was associated with an absence of novelty-induced cFos expression in dorsal CA1 (Fig. 2E), in L-NG hM4Di expressing animals compared to mCherry controls. We next tested whether a stimulation of ascending VN activity could compensate the effect of the age-associated GM on hippocampal function. To test this possibility, L-NG of YinY and AinY mice were transduced to express the excitatory DREADD (Fig. 2F). Three weeks later, intraperitoneal injection of a single dose of CNO (Fig. S2D) resulted in the induction of cFos 
expression in the L-NTS of the hM3Dq-transduced GM groups in comparison to mCherry-only control animals (Fig. S2E), demonstrating the robustness of the protocol used to activate the ascending VN pathway, irrespectively of the GM donor's age. In the ISO-NOL memory task, we showed that hM3Dq L-NG transduced AinY mice depicted a complete rescue of their memory abilities, when compared to AinY and YinY, mCherry controls (Fig. 2G). Noteworthy is the fact that a similar L-NG DREADD activation in YinY mice was not associated to any promemory effect in the same behavioral task (Fig. S2F). This suggests that pharmacogenetic VN stimulation can reinstate normal cognitive status rather than have a procognitive effect per se. At a cellular level, this DREADD ascending-VN stimulation also increased hippocampal cFos induction following animal exposure to novelty in L-NG hM3Dq transduced AinY mice when compared to AinY and YinY, mCherry-only expressing animals (Fig. $2 \mathrm{H}$ ).

Finally, we tested the effects of pharmacogenetic activation of L-NG on aged mice. The L-NG of 18-months old animals was transduced with the excitatory hM3Dq form of DREADDs (or mCherry for control, Fig. 2I). Animals were first submitted to an initial ISO-NOL task (ISONOL\#1) where all animals had deficits in their memory abilities in the task, irrespectively of their viral treatment group (Fig. 2J). The next day, L-NG mCherry-control and hM3Dq animal were i.p. CNO injected and submitted to a second different ISO-NOL task (ISO-NOL\#2, Fig. 2I). All hM3Dq transduced animals showed an increase in their memory abilities during the second testing, whereas mCherry transduced animals did not perform statistically differently between the two memory testing sessions (Fig. 2J).

In conclusion, our study unravels a direct contribution of age-associated GM - of both murine and human origin - on mouse hippocampal function. The findings also highlight the low VN tone as a key mechanism in this aged-related phenomenon (Fig. S3). It opens innovative therapeutic approaches aimed at acting either, directly on the GM composition, or on VN activity, to alleviate the impact of aging on hippocampus-dependent memory. 


\section{Methods}

Study approval. Humans: FM (fecal matter) donors that matched the young and aged-age category were selected from the Institut Pasteur (IP) ICAReB plateform's healthy volunteer Diagmicoll and CoSImmGEn cohorts. The clinical research protocols for collecting and handling of biological material and associated clinical and biological information were reviewed and approved by a French ethical committee "comité de protection des personnes Ile de France 1" (Ref: 09-12179, Ref: 2010-dec 12483) and is compliant with European general data protection regulations (GDPR). Donors received an oral and written information about the research and written informed consent was obtained from all recruited human subjects. Animals: experiments were performed using adult (> 10-week-old) male RjOrl: SWISS mice purchased from Janvier labs (St Berthevin, France). Animals were housed under a $12 \mathrm{~h} / 12 \mathrm{~h}$ light/dark cycle, with dry food pellets and water accessible ad libitum, at the Pasteur Institute animal care facility, officially registered for experimental studies on rodents. All animal experiments were designed according to the European communities council directive of 24 November 1986 (86/609/EEC) and the European Union guidelines, to the $3 R^{\prime}$ s rules and were supervised by the French Ministry of Research, as well as reviewed and approved by the Animal Welfare Committee of the IP (project number: 2016-0023) and the "Service prevention des risques" (experimental protocol 17.029). 


\section{Author contributions}

DR designed, developed and managed the project, performed the experiments and wrote the manuscript, under the supervision of PML. HS performed 16S rRNA gene sequencing analysis with the help of SS. MH and AHR were involved in behavioral and histological analysis. The ICAReB platform handled the donor samples regulatory and logistic aspects of the project under MNU supervision. 


\section{Acknowledgments}

The authors thank the study participants, physicians (Joëlle Brachat, Joël Ankri, Bertrand Denis, Dany Vythilingum), clinical/research assistants (Caroline Roussel, Céline Nemeth) and health officers (Anaïs Perilhou) who helped conduct this study, Laurence Motreff and Marc Monod from the Biomics Platform, C2RT, IP, Paris, France, supported by France Génomique (ANR-10-INBS-09-09), the life insurance company "MTRL" and IBISA; Blanca Liliana Perlaza, Amina Ait Saadi, Linda Sangari, Sophie Chaouche, Remy Artus from ICAReB; Oriana Lavielle, Gabriel Lepousez, Kurt Sailor and Ilana Gabanyi for reading the manuscript. 


\section{References}

1. Morais LH, Schreiber HL, Mazmanian SK. The gut microbiota-brain axis in behaviour and brain disorders. Nat Rev Microbiol. Published online October 22, 2020:1-15.

2. Bravo JA, et al. Ingestion of Lactobacillus strain regulates emotional behavior and central GABA receptor expression in a mouse via the vagus nerve. Proc Natl Acad Sci. 2011;108(38):16050-16055.

3. Clarke $G$, et al. The microbiome-gut-brain axis during early life regulates the hippocampal serotonergic system in a sex-dependent manner. Mol Psychiatry. 2013; 18(6):666-673.

4. Erny $D$, et al. Host microbiota constantly control maturation and function of microglia in the CNS. Nat Neurosci. 2015;18(7):965-977.

5. Möhle L, et al. Ly6Chi Monocytes Provide a Link between Antibiotic-Induced Changes in Gut Microbiota and Adult Hippocampal Neurogenesis. Cell Rep. 2016;15(9):1945-1956.

6. Ogbonnaya ES, et al., Adult Hippocampal Neurogenesis Is Regulated by the Microbiome. Biol Psychiatry. 2015;78(4):e7-e9.

7. Siopi E, et al. Changes in Gut Microbiota by Chronic Stress Impair the Efficacy of Fluoxetine. Cell Rep. 2020;30(11):3682-3690.e6.

8. Rothhammer $\mathrm{V}$, et al. Microglial control of astrocytes in response to microbial metabolites. Nature. 2018;557(7707):724-728.

9. Li Y, et al. Age-related shifts in gut microbiota contribute to cognitive decline in aged rats. Aging. 2020;12(9):7801-7817.

10. Thevaranjan $\mathrm{N}$, et al. Age-Associated Microbial Dysbiosis Promotes Intestinal Permeability, Systemic Inflammation, and Macrophage Dysfunction. Cell Host Microbe. 2017;21(4):455-466.e4.

11. Dahan L, Rampon C, Florian C. Age-related memory decline, dysfunction of the hippocampus and therapeutic opportunities. Prog Neuropsychopharmacol Biol Psychiatry. 2020;102:109943.

12. D'Amato A, et al. Faecal microbiota transplant from aged donor mice affects spatial learning and memory via modulating hippocampal synaptic plasticity- and neurotransmission-related proteins in young recipients. Microbiome. 2020;8(1):140.

13. Fülling C, Dinan TG, Cryan JF. Gut Microbe to Brain Signaling: What Happens in Vagus.... Neuron. 2019; 101(6):998-1002.

14. Altschuler SM, et al. Viscerotopic representation of the upper alimentary tract in the rat: sensory ganglia and nuclei of the solitary and spinal trigeminal tracts. J Comp Neurol. 1989;283(2):248-268.

15. Milby $\mathrm{AH}$, Halpern $\mathrm{CH}$, Baltuch $\mathrm{GH}$. Vagus nerve stimulation for epilepsy and depression. Neurother J Am Soc Exp Neurother. 2008;5(1):75-85.

16. Broncel $A$, et al. Medial septal cholinergic mediation of hippocampal theta rhythm induced by vagal nerve stimulation. PLoS ONE. 2018;13(11).

17. Sanders $\mathrm{TH}$, et al. Cognition-Enhancing Vagus Nerve Stimulation Alters the Epigenetic Landscape. J Neurosci. Published online February 25, 2019:2407-2418.

18. Bartosch S, et al. Characterization of Bacterial Communities in Feces from Healthy Elderly Volunteers and Hospitalized Elderly Patients by Using Real-Time PCR and Effects of Antibiotic Treatment on the Fecal Microbiota. Appl Environ Microbiol. 2004;70(6):35753581.

19. Claesson MJ, et al. Gut microbiota composition correlates with diet and health in the elderly. Nature. 2012;488(7410):178-184. 
20. Mariat $D$, et al. The Firmicutes/Bacteroidetes ratio of the human microbiota changes with age. BMC Microbiol. 2009;9:123.

21. Zwielehner J, et al. Combined PCR-DGGE fingerprinting and quantitative-PCR indicates shifts in fecal population sizes and diversity of Bacteroides, bifidobacteria and Clostridium cluster IV in institutionalized elderly. Exp Gerontol. 2009;44(6):440-446.

22. Mumby DG, et al. Hippocampal damage and exploratory preferences in rats: memory for objects, places, and contexts. Learn Mem Cold Spring Harb N. 2002;9(2):49-57.

23. Vogel-Ciernia A, Wood MA. Examining Object Location and Object Recognition Memory in Mice. Curr Protoc Neurosci Editor Board Jacqueline N Crawley Al. 2014;69:8.31.18.31.17.

24. Murai $T$, et al. Characteristics of object location memory in mice: Behavioral and pharmacological studies. Physiol Behav. 2007;90(1):116-124.

25. McEchron MD, et al. Hippocampectomy disrupts auditory trace fear conditioning and contextual fear conditioning in the rat. Hippocampus. 1998;8(6):638-646.

26. Bunzeck $\mathrm{N}$, et al. Pharmacological dissociation of novelty responses in the human brain. Cereb Cortex N Y N 1991. 2014;24(5):1351-1360.

27. Lisman JE, Grace AA. The Hippocampal-VTA Loop: Controlling the Entry of Information into Long-Term Memory. Neuron. 2005;46(5):703-713.

28. Yeoman $M$, Scutt $G$, Faragher R. Insights into CNS ageing from animal models of senescence. Nat Rev Neurosci. 2012;13(6):435-445.

29. Fung TC, Olson CA, Hsiao EY. Interactions between the microbiota, immune and nervous systems in health and disease. Nat Neurosci. 2017;20(2):145-155.

30. Carreno FR, Frazer A. Vagal Nerve Stimulation for Treatment-Resistant Depression. Neurotherapeutics. 2017;14(3):716-727. 


\section{Display items}

A

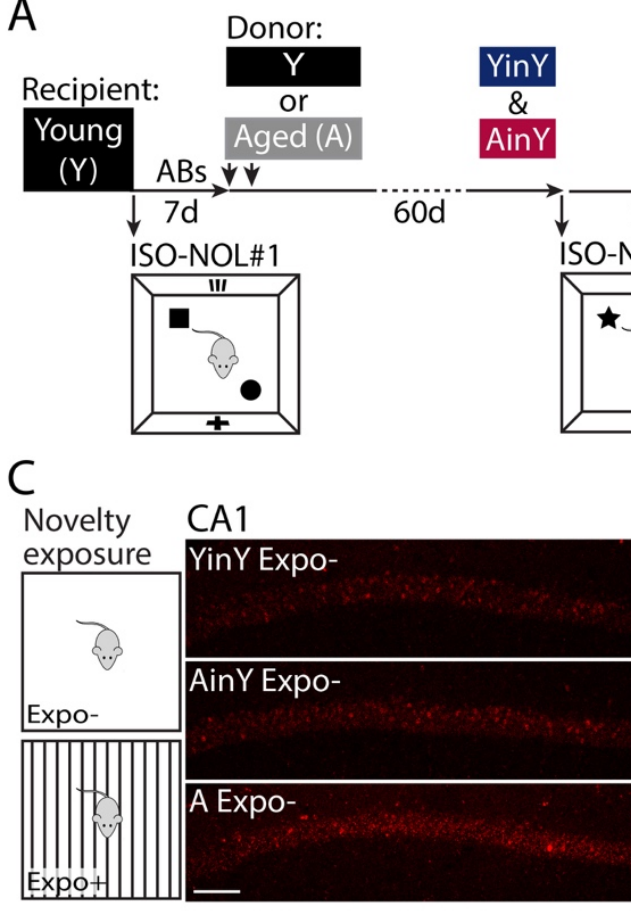

B
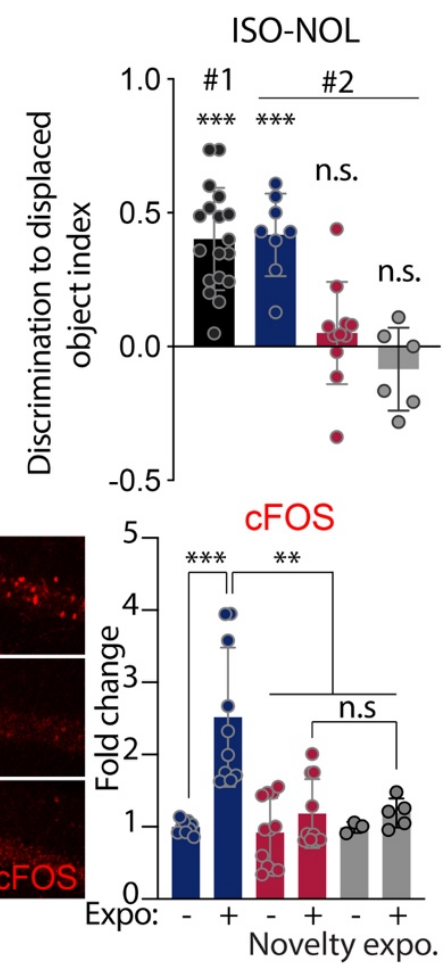

D

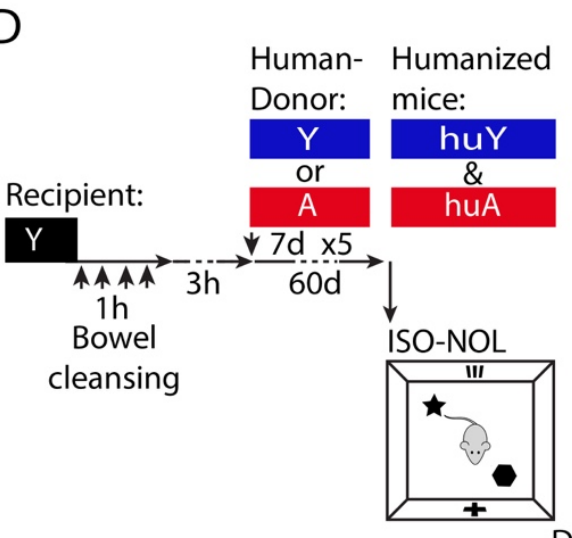

E

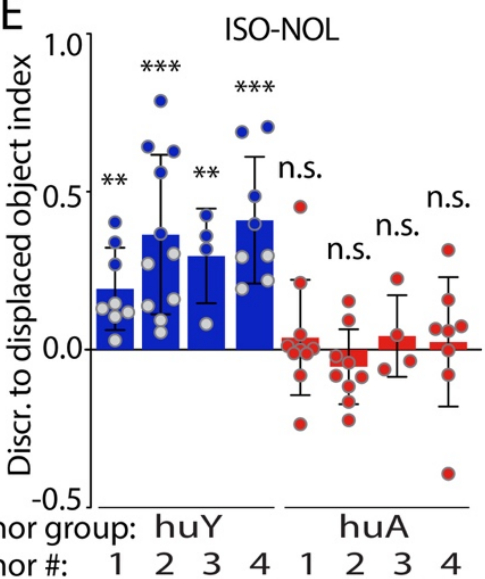

$\mathrm{F}$

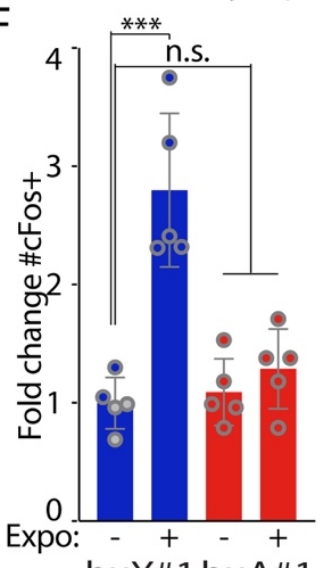

Fig. 1. Age-associated GM impairs hippocampal function and structure. (A) Schematic of the age-associated GM transfer scheme and behavior in young (Y) adult (2-3 months) mice and, (B) of its effect on memory abilities in the isotropic version of the novel object location task (ISO-NOL) of young adult mice prior to, and after, GM colonization from either young (Young mice GM in Young mice $=$ YinY blue bars) or aged ( $>18$ months) animals (Aged mice GM in young mice $=$ AinY) compared to aged mice ( $n=18,8,10$ and 6 mice per group). (C) Representative immunohistochemical images and quantitative analysis of the effect of the aging, versus young adult, -associated GM transfer compared to aged mice on CA1 increase number of cFos-positive cells after exposure to novelty ( $n=7,8,10,7,3$ and 5 mice per group). (D) Schematic of the human GM-transfer scheme and behavior in young adult mice. (E) Effect of the colonization of young adult mice with the GM from young human (huY) versus aged (HuA) donors on memory abilities in the ISO-NOL behavioral task ( $\mathrm{n}=4$ donors per age human-GM group, with 5 to 10 mice replicates per donor). (F) Quantification of the effect of huA donor\#1 versus huY donor\#1 human-GM transfer on recipient-mice's number of dorsal hippocampal CA1 cFos-positive cells after exposure to novelty ( $n=5$ per group). (B, E) one-tailed t test for each group and, (C, F) one-way ANOVA. Throughout the figure, bars represent the mean \pm SD. N.s., non-significant; $p>0.05 ;{ }^{*} p \leq 0.05 ;{ }^{* *} p \leq 0.01 ;{ }^{* * *} p$ $<0.001$. Scale bars, $100 \mu \mathrm{m}$. 


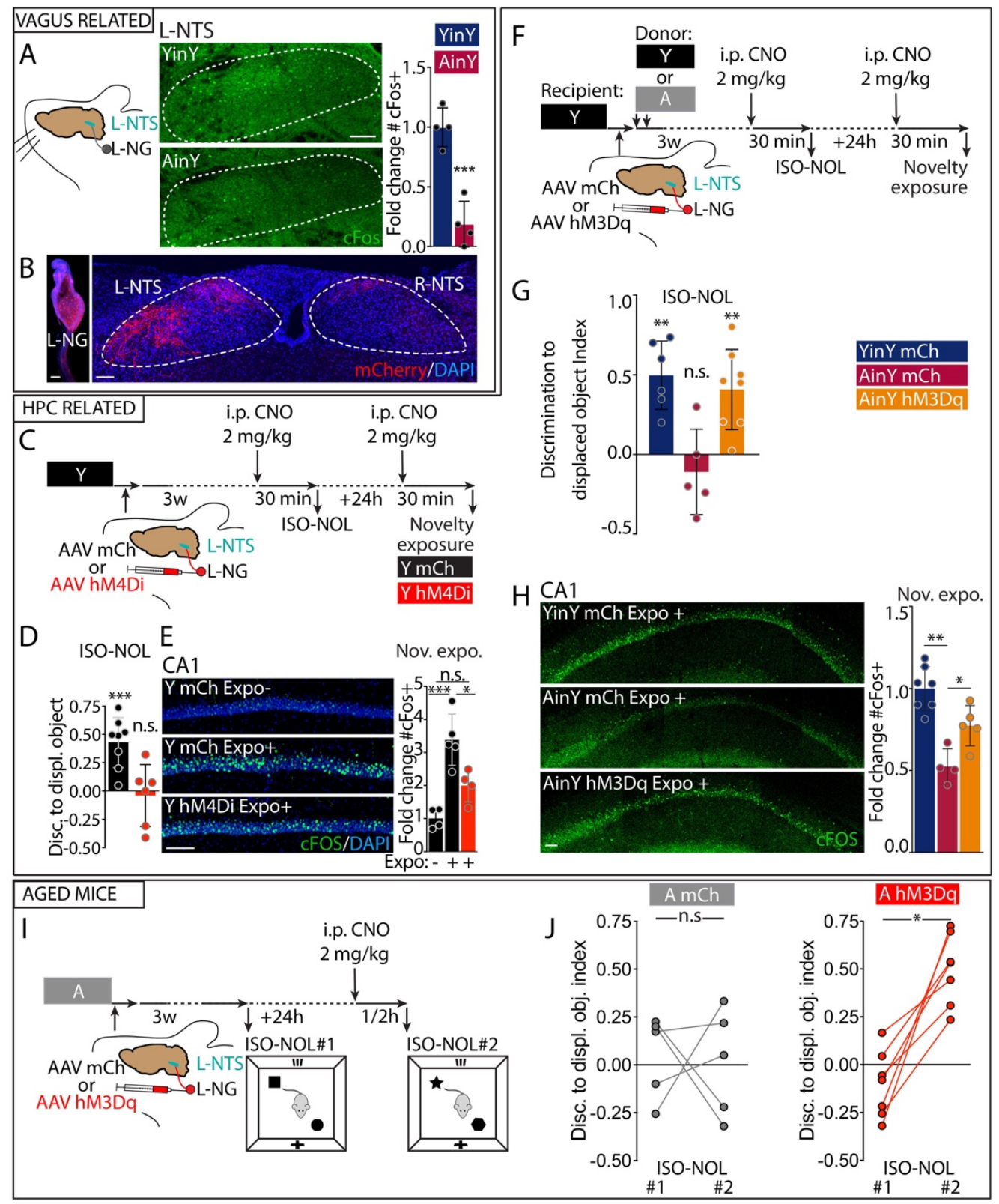

Fig. 2. Decrease in VN signaling is necessary and sufficient for aged-GM negative impact on memory and activation of VN ascending signaling increases memory abilities in aged mice. (A) Schematic of left nodose ganglia (L-NG) projections to the left nucleus of the solitary tract (L-NTS), and effect of aged-GM transfer on the number of cFos positive cells in the NTS ( $n=4$ per group). (B) Representative image of an AAV-mCherry transduced left nodose ganglia and of its ascending mCherry-positive fibers in the L- and right (R) -NTS. (C, F and I) Schematic of the viral and chemogenetic-based strategy to modulate VN ascending signaling in young $(C, F)$ and aged (I) mice where AAV5-hSyn-DIO-mCherry or hM4Di-mCherry (C) or hM3Dq-mCherry (F, I), was L-NG coinjected with an AAV1-hSyn-Cre (not represented), and timing for the behavior and pharmacogenetic ascending VN activity modulation. Effect of L-NG DREADD inhibition on, (D) the memory ability in the ISO-NOL task ( $n=8$ and 6) in L-NG hM4Di inhibited versus control mice and, (E) the number of dorsal hippocampal CA1 cFos-positive cells after exposure to novelty in L-NG hM4Di inhibited mice exposed to novelty versus mCherry-only control mice exposed or not to novelty ( $n=4,5$ and 4 per group). Effect of the chemogenetic vagal activation in AinY mice and its effect on, $(G)$ memory abilities in the novel object location task $(n=6,7,5$ and 8$)$ and, $(H)$ the number of dorsal hippocampal CA1 cFos-positive cells after exposure to novelty in YinY and AinY mCherry-only versus AinY hM3Dq expressing mice ( $n=7,4$ and 5 per group). (J) Effect of L-NG DREADD activation on ISO-NOL memory ability in L-NG mCherry or hM3Dq expressing aged mice, without and with ascending VN activation (n $=5$ and 7, two-tailed $t$ test with Wilcoxon matched-pairs signed rank test). (A) Student's $t$ test, $(D, G)$ one-tailed $t$ test for each group, $(E, H)$ one-way ANOVA. Throughout the figure, bars represent the mean \pm SD. N.s., nonsignificant; $p>0.05 ; *<0.05 ; * * p>0.01 ; * * p>0.001$. Scale bars, $100 \mu \mathrm{m}$. 